\title{
Incidental diagnosis and therapeutic approach of an iatrogenicintra- parenchymal pulmonary intercostal artery pseudoaneurysm: a case report
}

\author{
Giovanni Rossi ${ }^{1}$, Mario Petrillo ${ }^{2}$, Martina Flora ${ }^{3}$, Adriano Costigliola ${ }^{3}$, Mariano Mollica ${ }^{3}$, \\ Carlo Iadevaia $^{3}$, Giampiero Gaio ${ }^{4}$, Francesco S. Cerqua ${ }^{3}$, Andrea Bianco ${ }^{3}$, Fabio Perrotta ${ }^{5}$ \\ ${ }^{1}$ Department of Radiology, Monaldi Hospital, Naples; ${ }^{2}$ Department of Diagnostic and Interventional Radiology, San \\ Paolo Hospital, Department of Health Science, University of Milan; ${ }^{3}$ Department of Translational Sciences, University \\ of Campania "L. Vanvitelli", Naples; ${ }^{4}$ Adult Congenital Heart Disease Unit, Monaldi Hospital, Naples; ${ }^{5}$ Department of \\ Medicine and Health Sciences "V. Tiberio", University of Molise, Campobasso, Italy
}

\begin{abstract}
Intercostal artery pseudoaneurysms (IAP) are extremely rare but its sudden rupture represents a life-threatening complication. We report an unusual case of a late intercostal artery pseudoaneurysm, after a video-assisted thoracoscopic surgery, presenting as a large intra-parenchymal lung mass. Diagnosis was made by biphasic computed tomography angiography and contrastenhanced pulsed-wave doppler ultrasound. To minimize the risk of aneurysm bleeding immediate angiographic embolization was planned and successfully performed. IAP should be considered in presence of lung mass in patients who have previously undergone an intervention with intercostal approach to prevent to prevent lifethreatening complications.
\end{abstract}

\section{Introduction}

Intercostal artery pseudoaneurysm (IAP) is a rare complication of surgical procedures involving intercostal space. It is generally

Correspondence: Fabio Perrotta, Department of Medicine and Health Sciences "Vincenzo Tiberio", University of Molise, Campobasso, 86100 Italy. Tel. +39.0874.4041. E-mail: fabio.perrotta@unimol.it

Key words: Intercostal artery pseudoaneurysm, iatrogenic pseudoaneurysm, embolization; CT angiography.

Conflict of interest: The authors declare no competing of interest in association with this manuscript.

Received for publication: 14 May 2019.

Accepted for publication: 11 July 2019.

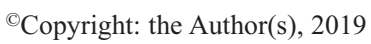

Licensee PAGEPress, Italy

Monaldi Archives for Chest Disease 2019; 89:1094

doi: 10.4081/monaldi.2019.1094

This article is distributed under the terms of the Creative Commons Attribution Noncommercial License (by-nc 4.0) which permits any noncommercial use, distribution, and reproduction in any medium, provided the original author(s) and source are credited. asymptomatic and difficult to diagnose until its rupture. Sudden bleeding may occur in IAP, generally into pleural space or retroperitoneum, with a rapid decline in cardiopulmonary status, with high risk of mortality in absence of treatment $[1,2]$.

In presence of comorbidity, symptoms may be aspecific reflecting different phenotypes of underlying diseases [3-7]. We present the first report in literature of a late onset intercostal artery pseudo aneurysm following video-assisted thoracoscopic surgery (VATS), presenting as large intra-parenchymal mass. Among diagnostic procedures imaging is relevant for differential diagnosis of lung intra-parenchyma mass and CT angiography is crucial to characterize vascular involvement [8-12]. Following diagnosis by biphasic computed tomography angiography and contrast-enhanced pulsed-wave doppler ultrasound study, an immediate angiographic embolization was planned and successfully performed to minimize the risk of aneurysm bleeding.

\section{Case Report}

An 82-year-old male, ex-smoker with asbestos exposure, with clinical history of pacemaker implantation for trifascicular heart block in 2016 and COPD presented with one month of exertional dyspnoea unresponsive to pharmacological treatments. He had clinical history of pleuritis in fibrotic evolution, diagnosed by VATS biopsy performed during diagnostic procedure for parenchymal consolidation associated with pleural effusion in 2014.

The Chest X-Ray (CXR) revealed a large opacity in the right lower lobe. A Chest Computed Tomography (CT) was performed, showing presence of a non-homogeneous well capsulated pulmonary mass $(6 \times 5.5 \mathrm{~cm})$ (Figure 1a). No alteration in blood chemistry and a mild reduction of alveolar capillary diffusion capacity was observed. A percutaneous CT-guided fine-needle aspiration biopsy (FNAB) detected the presence of haematic material. Following radiologic case review, a biphasic CT angiography was performed; the pre-surgical chest CT was analysed and a tiny colliquation nuclei in the fibrotic collapsed parenchyma adjacent to the pleural chronic thickening was identified (Figure 1b). A biphasic CT angiography was then performed on pulmonary and systemic vessel which showed the vascular nature of the mass: a network of tubular vessels, surrounded by extensive clot and hyperdense walls. The diagnosis of a chronic pseudo-aneurysm was finally made (Figure $1 \mathrm{c}, \mathrm{d}$ ). To determine the systemic or pulmonary feeding of the mass, a 
contrast-enhanced pulsed-wave doppler ultrasound study (PWDUS) was performed, which confirmed the prevalence of systemic origin of the vessels (Figure 2 a,b). Patient underwent digital subtraction angiography (DSA) that detailed the presence of a pseudoaneurysm arising from XII right intercostal artery and draining into the inferior lobar vein (Figure 2c). The embolization of the XII right intercostal artery was performed, across femoral access, using two coils of Microplex10 5/22 mm and 5/6 mm, respectively. There were no complications in the execution of the embolization and in post-operative period. Post embolization angiography showed successful occlusion of the pseudoaneurysm (Figure 2d).

After one month, the patient reported an improvement in respiratory symptoms and $\mathrm{CT}$ angiography examination showed a marked reduction of intralesional vessels. Follow-up visits continued with functional examinations and Tc-scan that showed stability in both the respiratory symptoms and radiological scan, up to the last 2-year check-up from the interventional procedure.

\section{Discussion}

A growing body of researches reported that VATS and minimally invasive surgical techniques have a worthy safety profile in both benign and malignant thoracic diseases [13-16]. IAP is extremely rare with only few cases reported in the literature to our knowledge. The aetiology has been reported to be traumatic in two $[17,18]$ and iatrogenic in eleven cases due to procedures involving an intercostal approach [1,2,19-21]. VATS procedure was identified cause of IAP only in one case in literature [19]. In our case the lesion vessel appeared to emerge very close to pleural biopsy site. We can hypothesize that the injury was made by contact of the VATS instruments or drainage tubes with visceral pleural vessels. The late onset of the pseudoaneurysm formation is confirmed by a negative post-operative CT scan performed nine months after VATS. In most of the cases IAP presented within one month of the procedure. We incidentally diagnosed IAP two years
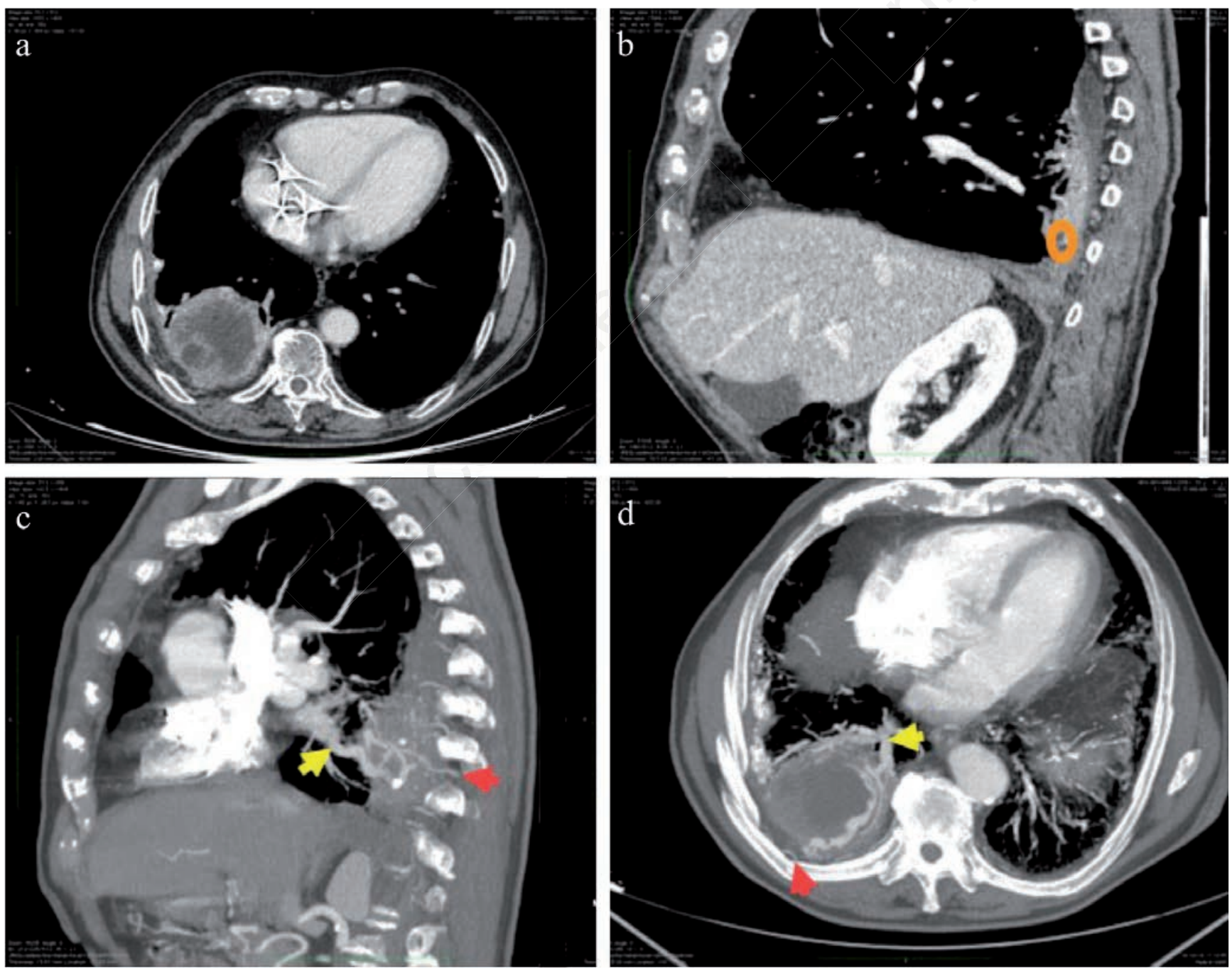

Figure 1. a) Axial contrast-enhanced CT scan. Heterogeneous well capsulated pulmonary mass is seen in inferior right lobe $(6 \times 5.5 \mathrm{~cm})$. b) Sagittal chest CT shows parenchyma consolidation with proximal air bronchogram, tiny calcified parenchyma and pleural nodule and a colliquative intra-parenchyma fluid loculation (orange circle) and organized pleural effusion is also evident. c,d) Sagittal and Axial Maximum Intensity Projection (MIP) of the CT Angiography shows a network to tubular vessels, rounded by extensive cloth of and hyperdense wall: XII intercostal artery (red arrow) and pulmonary vein (yellow arrow) are evident. 
after the intervention of VATS, during investigations for exertional dyspnoea.

Haemothorax, due to the bleeding of pseudoaneurysm into pleural space, is the most common clinical presentation; atypical onset with a pulsatile mass [19,22], an extrapleural hematoma [23] and a retroperitoneal haematoma [24] have also been reported in literature.

To our knowledge this is the first case of large tumour-like intraparenchymal mass presentation of the pseudoaneurysm. Our patient presented with exertional dyspnoea in part due to underlining COPD without haemoptysis nor fatigue.

Due to the risk of early bleeding after its formation, diagnosis and treatment before rupture is critical. Definitive diagnosis of IAP is usually made by DSA [20-22], but CT and US can be very useful as primary diagnostic modality $[21,22,25]$. In our case, a biphasic CT angiography on pulmonary and systemic vessel was fundamental to recognize the vascular nature of the mass and make the diagnosis. The imaging showed the typical radiological findings of intraparenchymal IAP: a network of tubular vessels surrounded by extensive clot and hyperdense walls of collapsed lung. Furthermore, we used contrast-enhanced PWDUS for the first time on IAP to confirm the systemic origin of feeding vessels and guide the therapy.

Embolization with metallic or microcoils is considered the first line treatment [26]. There are few reports of treatment with covered stents [27], percutaneous thrombin injections [28], post embolization [21] or aneurismectomy [27] as first line of treatment and two cases of conservative management $[25,28]$. We successfully treated our patient with embolization of the XII right intercostal artery using two coils of Microplex100 5/22 mm and 5/6 mm, respectively.

\section{Conclusions}

Intercostal artery pseudoaneurysm should be considered in presence of lung mass in patients who have previously undergone
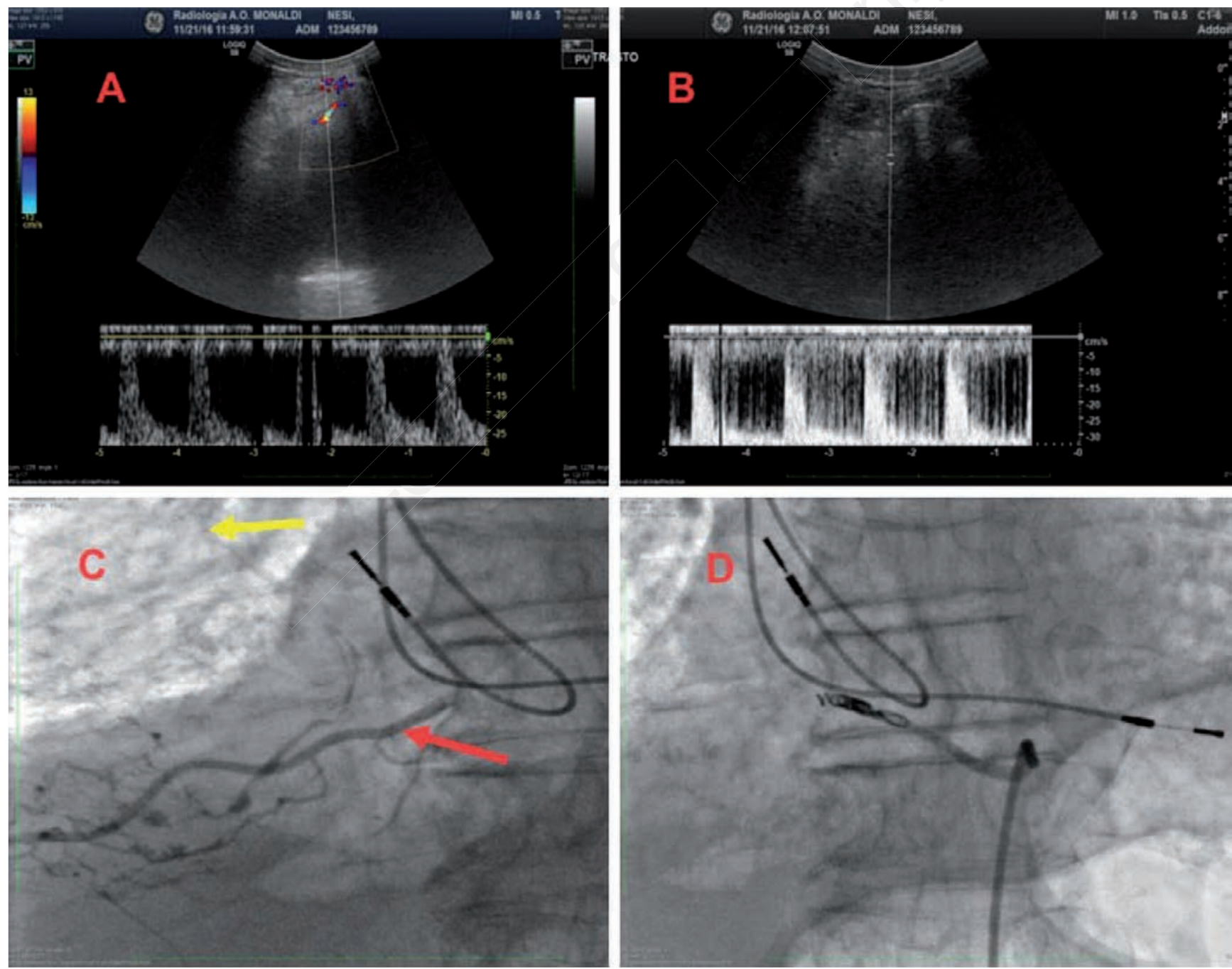

Figure 2. a) Ultrasound study of the pseudo-aneurysm feeding vessel; the color Doppler ultrasound hardly shows the signal of the feeding vessel. b) The contrast-enhanced power wave Doppler ultrasound clearly shows the typical systemic spectral feature of vessel; c) Digital subtraction angiography (DSA); the DSA, due to its better spatial resolution, clearly evidences the thin network of intraparenchyma vessels (red arrow); in the static imagine it is hardly evident the draining pulmonary vein (yellow arrow). d) Post-embolization DSA evidences absence of flow of the intercostal feeding artery distal to micro-coils. 
an intervention with intercostal approach. CT angiography is essential to the diagnosis in these patients and contrast-enhanced PWDUS can guide the subsequent minimally invasive treatment. Early angiographic treatment should be carried out as emergency procedure after diagnosis of IAP to prevent life-threatening complications.

\section{References}

1. Aoki T, Okada A, Tsuchida M, Hayashi J. Ruptured intercostal artery pseudoaneurysm after blunt thoracic trauma. Thorac Cardiovasc Surg 2003;51:346-7.

2. Sekino $\mathrm{S}$, Takagi $\mathrm{H}$, Kubota $\mathrm{H}$, et al. Intercostal artery pseudoaneurysm due to stab wound. J Vasc Surg 2005;42: 352-6.

3. Maniscalco M, Bianco A, Mazzarella G, Motta A. Recent advances on nitric oxide in the upper airways. Review. Current Med Chem 2016;23:2736-45.

4. Bianco A, Nigro E, Monaco ML, et al. The burden of obesity in asthma and COPD: Role of adiponectin. Pulm Pharmacol Ther 2017;43:20-5.

5. Nigro E, Daniele A, Scudiero O, et al. Adiponectin in asthma: implications for phenotyping. Curr Protein Pept Sci 2015; $16: 182-7$.

6. Mazzarella G, Iadevaia C, Guerra G, et al. Intralobar pulmonary sequestration in an adult female patient mimicking asthma: a case report. Int J Surg 2014;12:S73-7.

7. Masiello R, Iadevaia C, Grella E, et al. A case of multiple unilateral pulmonary arteriovenous malformation relapse: Efficacy of embolization treatment. Open Med (Wars) 2015;10:513-8.

8. Brunese L, Greco B, Setola FR, et al. Non-small cell lung cancer evaluated with quantitative contrast-enhanced CT and PET-CT: net enhancement and standardized uptake values are related to tumour size and histology. Med Sci Monit 2013;19:95-101.

9 . Maniscalco M, Vitale C, Vatrella A, et al. Fractional exhaled nitric oxide-measuring devices: technology update. Med Devices (Auckl) 2016;9:151-60.

10. de Blasio F, Di Gregorio A, de Blasio F, et al. Malnutrition and sarcopenia assessment in patients with chronic obstructive pulmonary disease according to international diagnostic criteria, and evaluation of raw BIA variables. Respir Med 2018; 134:1-5.

11. Mazzarella G, Lucariello A, Bianco A, et al. Exposure to submicron particles (PM1.0) from diesel exhaust and pollen allergens of human lung epithelial cells induces morphological changes of mitochondria tonifilaments and rough endoplasmic reticulum. In Vivo 2014;28:557-61.

12. De Blasio F, de Blasio F, Miracco Berlingieri G, et al. Evaluation of body composition in COPD patients using multifrequency bioelectrical impedance analysis. Int J Chron Obstruct Pulmon Dis 2016;11:2419-26.

13. Perrotta F, Cerqua FS, Cammarata A, et al. Integrated therapeutic approach to giant solitary fibrous tumor of the pleura: report of a case and review of the literature. Open Med (Wars) 2016;11:220-5.

14. Amore D, Mazzella A, Izzo A, et al. Management of pericardial cyst in the mediastinum: a single-port approach. J Bras Pneumol 2016;42:302-3.

15. Mazzella A, Izzo A, Amore D, et al. A new perspective on the treatment of complicated giant emphysematous bulla. A case report. Ann Ital Chir 2016;87 (ePub) pii: S2239253X16024816.

16. Mazzella A, Izzo A, Amore D, et al. Single port VATS resection of a sessile solitary fibrous tumour of the visceral pleura. A case report. Ann Ital Chir 2015;86 (ePub) pii: S2239253X15024457.

17. Long SS, Johnson PT, Fishman EK. Intercostal artery pseudoaneurysm due to thoracentesis: diagnosis with threedimensional computed tomographic angiography. J Comput Assist Tomogr 2012;36:100-2.

18. Gutierrez Romero DF, Barrufet M, Lopez-Rueda A, Burrel M. Ruptured intercostal artery pseudoaneurysm in a patient with blunt thoracic trauma: diagnosis and management. BMJ Case Rep 2014 pii: bcr2013202019.

19. Atherton WG, Morgan WE. False aneurysm of an intercostal artery after thoracoscopic sympathectomy. Ann R Coll Surg Engl 1997;79:229-30.

20. Melloni G, Bandiera A, Crespi G, Zannini P. Intercostal artery pseudoaneurysm after computed tomography-guided percutaneous fine needle aspiration lung biopsy. J Thorac Imaging 2012;27:W48-9.

21. Kawai H, Ito M. Intercostal artery pseudoaneurysm after thoracoscopic lung resection. Gen Thorac Cardiovasc Surg 2009;57:550-5.

22. Alonso SF, Azcona CM, Heredero AF, de Cubas LR. Poststernotomy intercostal artery pseudoaneurysm. Sonographic diagnosis and thrombosis by ultrasound-guided percutaneous thrombin injection. Interact Cardiovasc Thorac Surg 2009; 9:722-4.

23. Rodriguez M, González de la Huebra Labrador T, Aranda J. Diagnosis and treatment of hemothorax caused by intercostal artery pseudoaneurysm. Arch Bronconeumol 2016;52:219.

24. Callaway MP, Wilde P, Angelini G. Treatment of a false aneurysm of an intercostal artery using a covered intracoronary stent-graft and a radial artery puncture. Br J Radiol 2000;73: 1317-9.

25. Bluebond-Langner R, Pinto PA, Kim FJ, Hsu T, Jarrett TW. Recurrent bleeding from intercostal arterial pseudoaneurysm after retroperitoneal laparoscopic radical nephrectomy. Urology.60:1111, 2002.

26. Briganti F, Leone G, Marseglia M, et al. Endovascular treatment of cerebral aneurysms using flow-diverter devices: A systematic review. Neuroradiol J 2015;28:365-75.

27. Briganti F, Napoli M, Leone G, et al. Treatment of intracranial aneurysms by flow diverter devices: long-term results from a single center. Eur J Radiol 2014;83:1683-90.

28. Yamakado K, Nakatsuka A, Tanaka N, et al. Transcatheter arterial embolization of ruptured pseudoaneurysms with coils and n-butyl cyanoacrylate. J Vasc Interv Radiol 2000;11:66-72. 